\title{
CONSIDERAÇÕES SOBRE O ESTADO DE SAUDE DA CRIANÇA
}

\author{
Giselle Dupas *
}

DUPAS, G. Consideraçōes sobre o estaao de saúde da criança. Rev. Esc. Enf. USP., v. 27, n. 3, p. 362-71, dez. 1993.

Os indicadores de saude, como oa coaficientes de morbidade e mortalidade infantis, devem ser analisados a fim de se estabelecer prioridades para a assistência à saúde da criança. Observa-se na literatura que a Taxa de Mortalidade Infantil de Menores de 5 anos (TMM 5) vem aumentando no Brasil, nos últimos tempos, principalmente em decorrência das deficiências nutricionais e das doenças infecciosas, apesar do estabelecimento de programas e metas instituídos com o objetivo de diminuir essa incidência.

UNITERMOS: Saúde da criança. Criança. Morbi-mortalidade infantil.

\section{INTRODUÇÃO}

É sabido que a assistência à saúde não se encontra igualmente acessível para todas as crianças. Embora seja de inquestionável importância, é escasso em nosso meio, o conhecimento sobre a cobertura e a qualidade da assistência materno-infantil. A inexistência de um sistema único de saúde e a precariedade com que os múltiplos serviços existentes registram suas atividades, sāo fatores que certamente contribuem para explicar o quadro crítico da situação de saúde encontrado (MONTEIRO, 1988).

Segundo BENICIO et al (1992), fica cada vez mais delineada a importância do conhecimento das necessidades de saúde dos diversos grupos populacionais, expressas através de indicadores epidemiológicos, bem como a avaliação do acesso desses mesmos grupos aos serviços de saúde. Em nosso meio, e em vários outros países latino-americanos, ganha importância fundamental a questão da "grande desigualdade em saúde", tanto do ponto de vista epidemiológico, com perfis diferenciados

Enfermeira. Mestre em Enfermagem, Assistente do Departamento de Enfermagem e Obstetricia da Universidade Federal de Sào Carlos. 
de morbi-mortalidade dos diferentes grupos sociais, como também no que diz respeito à grande diferença de acesso e uso dos serviços de saúde.

Ainda que sejam apontados neste texto alguns dados sobre morbimortalidade infantil, não foi nosso objetivo realizar uma revisảo bibliográfica sobre o assunto. Antes, esses dados são apresentados a fim de fornecer subsídios para iniciarmos a reflexão sobre a relação deles com as deliberaçōes e programas criados para atender a população infantil, bem como o quanto suas metas e objetivos têm se efetivado.

\section{DADOS DA REALIDADE ATUAL}

O estudo do homem e seu processo evolutivo sempre foram alvo de preocupação e investigação, sendo que atualmente o período da infância vem recebendo ênfase marcante por parte de organizaçōes governamentais e certas entidades, nacionais e internacionais.

Esta preocupação está sujeita a visōes diversificadas. Para muitos, quando se fala em assistência à criança, aparecem imagens de grandes construções, bem equipadas, com pessoal altamente qualificado, em centros urbanos desenvolvidos; para outros, delineia-se a imagem da criança no convívio familiar e comunitário, com problemas de ordem social, tais como moradia, saneamento, alimentação, educação e recreação.

Sabemos que o cuidado para com esse "ser", que representa a perspectiva futura da nação e da humanidade, deve abranger os níveis primário, secundário e terciário de assistência, e não se ater a uma única forma de assistência. Deve-se partir dos problemas que mais acometem a comunidade infantil.

Uma das formas de analisarmos o nível de assistência a ser priorizado, conforme GODOY (1984), seria através da observação de indicadores de saúde, tais como os coeficientes de morbidade e de mortalidade infantis, entre outros.

Segundo MORA et al (1986) e PAIM et al (1987), falar em morbidade e mortalidade infantil, no Brasil, assim como na maioria dos países do chamado terceiro mundo é tarefa difícil. A própria Organização Panamericana de Saúde admite a infidelidade desses coeficientes e de outros, devido à dificuldade primeiro em encontrar todos os dados registrados $\mathrm{e}$ segundo, na pouca confiabilidade dos mesmos (ASPECTOS, 1987). Por exemplo, estudo realizado no Brasil pela OPAS (ASPECTOS, 1987), mostrou que $20 \%$ ou mais do número de óbitos haviam sido registrados como "sinais, sintomas e estados mórbidos mal definidos". Esse tipo de dado reflete a deficiência no registro de dados, o que dificulta a investigação sobre as patologias que acometem as crianças, e que têm como conseqüência o óbito.

Documentos da OPAS (1988) e do FUNDO DAS NAÇÕES UNIDAS PARA A INFÂNCIA - UNICEF - (1991) trazem uma análise do coeficiente 
de mortalidade infantil: diariamente, 40.000 crianças menores de 5 anos morrem no mundo em desenvolvimento; dessas, aproximadamente 14 milhöes morrem anualmente, antes de completarem o primeiro ano de vida. Observamos, pelos relatórios de anos anteriores, que esses números vêm aumentando significativamente.

O Brasil, que, no relatório da UNICEF 1990, estava incluído como um dos países com Taxa de Mortalidade Infantil de Menores de 5 anos (TMM 5) média (de trinta e um a noventa e quatro mortes para cada mil nascidos vivos), no relatório de 1991, aparece incluído como um dos países com TMM 5 alta (de setenta e um a cento e quarenta mortes para cada mil nascidos vivos).

Os governos membros da OPAS definiram uma meta mínima, estipulando que, no ano dois mil além de saúde para todos e uma taxa de 50 óbitos para cada mil nascidos vivos, a esperança de vida ao nascer não deverá ser inferior a 70 anos. Contudo, essa meta, que os países da América Latina devem alcançar entre os anos 2000-2005, tem o mesmo índice que existia na América do Norte, nos anos de 1958-1955 (ASPECTOS, 1987).

Para MORA et al (1986), o Brasil é um país com realidades contrastantes de região para regiāo. As taxas de mortalidade infantil (TMI) variam de uma zona para outra e até mesmo dentro de uma única cidade - zona urbana, zona urbana periférica e zona rural -. Estes contrastes sociais têm forte peso na determinação deste indicador social.

MONTEIRO et al (1986) detectaram, em estudo realizado no município de São Paulo, que as formas de desnutrição lá encontradas eram, em sua maioria, de primeiro grau, apresentando nítida tendência de elevação, à medida que se inferioriza o estrato sócio-econômico familiar. Este mesmo município situa-se em posição privilegiada em relação ao conjunto de populaçōes do Terceiro Mundo e inclusive em relação a outros municípios e regiōes do próprio Brasil, principalmente do Norte e Nordeste, embora ressaltando-se que na periferia deste município a condição sócio-econômica da população é bastante precária.

O relatório da UNICEF (1989) analisa também a alta porcentagem de crianças com baixo peso ao nascer, assim como seu grau de nutriçāo até 5 anos, mostrando a relação estreita entre crescimento físico/mental das crianças com o desenvolvimento sócio-econômico de suas sociedades.

$\mathrm{O}$ impacto adverso que vem tendo a recessão econômica atual não tem sido levado em consideração sobre este grupo da população. Segundo WILLIAMSON (1989), nem sempre existe uma correlação direta entre os níveis de desenvolvimento econômico e social de um país. Alguns países com elevado Produto Interno Bruto (PIB) têm um índice de qualidade de vida baixo, indicando que estão utilizando seus recursos financeiros de forma ineficientes.

Esses índices indicam que crescimento, desenvolvimento e saúde da criança, nos países sub-desenvolvidos, são afetados por deficiências nutricionais e doenças infecciosas, bem como por suas complicaçōes, for- 
mando um ciclo vicioso onde desnutrição e infeç̧ão, ficam ligados diretamente à pobreza. Cerca de 130 milhões de crianças menores de 5 anos da população dos países em desenvolvimento, estão sofrendo de desnutrição. As doenças diarréicas matam 4 milhōes de crianças por ano e mais de 2 milhōes morrem por insuficiência respiratória aguda (WILLIAMSON, 1989).

SAAD (1986) esquematiza, da seguinte forma, a situação de morbidade infantil no Brasil, mais especificamente no Estado de Sāo Paulo:

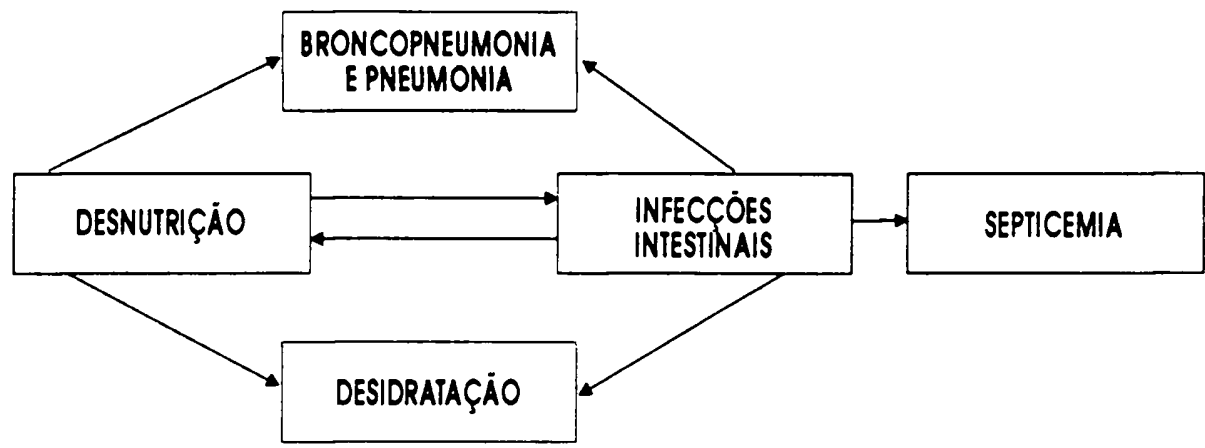

* Esquema adaptado de SAAD (1986)

As crianças que sobrevivem à desnutrição grave, além de terem freado seu crescimento físico, apresentam diversos graus de atraso mental devido ao efeito combinado do déficit alimentar crônico, das infeç̧ões repetidas e da privação psicoafetiva e social. Há uma diminuição da capacidade de aprendizagem, que se traduz num rendimento escolar baixo e posteriormente em um fracasso de sua capacitação como adulto. Mesmo quando a privação relatada for temporária, isso pode vir a acontecer.

\section{PROPOSTAS PÚBLICAS DE ASSISTÊnCIA A SAÚdE DA CRIANÇA}

Nos últimos vinte anos, sob o patrocínio do Estado autoritário, a atenção à saúde no Brasil passou por um acelerado processo de privatização, tendo como eixo a instituição hospitalar, à qual passou a incorporar um aparato tecnológico de crescente complexidade e sofísticação (NAKAMAE, 1987).

Entretanto, essa sofisticação não se encontrava, e tampouco se encontra atualmente, à disposição e nem atendem as necessidades da maioria da população, muito menos da população infantil, uma vez que 
os coeficientes de morbi-mortalidade têm aumentado com o passar do tempo, observando relação direta com a queda da qualidade de vida da população.

ALVARENGA (1991), coloca que no processo de crescimento e desenvolvimento, em função das características de ser imaturo e dependente, a criança é submetida a relaçōes sociais definidas, que passam a expressar a forma pela qual a sociedade cuida dos corpos, das mentes e das consciências, definindo assim, no interior de uma mesma sociedade, processos diferenciados, de acordo com sua inserção em classes sociais determinadas. Assim, problemas de natureza social, configuram-se como determinantes do crescimento e desenvolvimento infantil.

Corroborando com o exposto, MONTEIRO (1988) coloca que em sociedades de economia capitalista, os bens e serviços produzidos pelo conjunto da sociedade, incluindo os essenciais à sobrevivência, não são igualmente acessíveis a todos os indivíduos; antes dependem de sua inserção no processo social de produção, ou seja, de sua condição de classe. Em face desta situação, pode-se dizer que é a classe social de indivíduo aquela que, em última instância, irá determinar os limites mais prováveis dentro dos quais se definirá sua situaçāo no processo saúde/doença.

No que se refere a assistência à criança, ROCHA (1987) coloca que, embora no discurso a Puericultura (ciência que se ocupa em cultivar a vida e a saúde das crianças) afirme destinar-se a todas as crianças indistintamente, observa-se que retrata a diferenciaçāo em classe sociais, quando se concretiza. Portanto, há distintas "Puericulturas" destinadas a crianças de distintas classes sociais.

Segundo a mesma autora, existe um distanciamento entre o conceito doutrinário e a prática. No conceito doutrinário, a criança é entendida como um ser universal e abstrato, não incorporando as enormes diferenças de condições de vida que separam os diferentes extratos da população.

O Ministério da Saúde, com apoio de organizaçōes internacionais e de outros setores da sociedade, desenvolveu planos e estratégias para a extensāo de cobertura de serviços a toda a população, sendo que a assistência à criança teve como marco o Programa Materno Infantil, de 1974, e as Ações Básicas na Assistência Integral à Saúde da Criança, de 1984 (ROCHA et al, s/d).

Essa ação do Ministério da Saúde de 1984, baseou-se na análise das condições sanitárias da população, a fim de atender uma das parcelas mais significativas de usuários dos serviços de saúde, ou seja, a criança de classe social menos favorecida economicamente. Assim, o programa "Assistência Integral à Saúde da Criança" manifestou-se norteado por diretrizes que pretendiam desenvolver um acompanhamento sistemático do crescimento e desenvolvimento infantil, bem como resolver, a partir das unidades básicas de saúde, a maioria dos problemas de saúde da criança menor de cinco anos de idade (BRASIL, 1984). 
O objetivo mais amplo deste programa refere-se a redução da morbidade na faixa de 0 a 5 anos de idade, tendo como objetivos específicos:

- utilizar o acompanhamento do crescimento e desenvolvimento como metodologia para organização da assistência à criança nesta faixa etária;

- promover o aleitamento materno e orientar a alimentação no primeiro ano de vida;

- aumentar os níveis de cobertura vacinal de acordo com as normas técnicas do Ministério da Saúde;

- identificar precocemente os processos patológicos, favorecendo o diagnóstico e tratamento oportunos;

- promover a educação para a saúde, destacando a importância da participação da família nas atividades de assistência à criança.

Entretanto, por mais salutar que sejam os objetivos, os índices de morbi-mortalidade infantil continuam elevados. Acreditamos que is to se deva ao fato dos programas lançados pelo Ministério da Saúde serem totalmente desvinculados de programas de outros ministérios, que se proponham a melhorar a qualidade de vida da população, condição primeira para redução da morbidade.

Para FERREIRA et al (1984), a pediatria trata de pessoas essencialmente dependentes de fatores familiares e ambientais, de modo que é necessária uma intensa ação educativa sobre a família e a comunidade, a fim de garantir que orientações sejam dadas para facilitar o suprimento das necessidades da criança; necessidades biológicas e psicossociais específicas que, segundo CAMPESTRE (1983), são inerentes ao processo de crescimento e desenvolvimento humanos, predominantemente nos primeiros anos de vida.

Documentos da OPAS (1988) e da UNICEF (1991), tecem consideraçōes a respeito da ação educativa. A alfabetização materna tem sido apontada como instrumento valioso na redução da morbi-mortalidade infantil e como base do desenvolvimento da sociedade de modo geral. Por esse motivo, o primeiro Encontro Mundial de Cúpula pela Criança, realizado em Nova Iorque, em setembro de 1990, reunindo setenta e uma naçōes, dentre elas o Brasil, deliberou metas a serem atingidas no ano 2000, como:

- redução de um terço nas taxas de mortalidade infantil com relação a 1990, ou a nível de 70 por 1.000 nascidos vivos;

- redução de $50 \%$ nas taxas de mortalidade materna com relação a 1990; 
- redução de $50 \%$ nas taxas de desnutrição grave e moderada entre menores de cinco anos, com relação aos níveis de 1990;

- acesso universal à água limpa e a meios sanitários de eliminaçāo de dejetos;

- acesso universal à educação básica e à conclusão da escola primária por pelo menos $80 \%$ das crianças em idade escolar;

- redução de pelo menos $50 \%$ na taxa de analfabetismo de adultos com relação aos níveis de 1990 , com ênfase na alfabetização de mulheres;

- proteção para as crianças que vivem em circunstâncias particularmente difíceis, especialmente em situações de conflito armado.

\section{CONSIDERAÇÕES FINAIS}

Alguns dados encontrados sobre morbi-mortalidade infantil são contraditórios. Enquanto documentos da UNICEF (1990 e 1991) apontam uma estimativa de elevação do coeficiente de morbi-mortalidade no Brasil, documentos do Instituto Brasileiro de Geografia e Estatística (IBGE, 1992), declaram que diversos estudos têm mostrado uma clara tendência de queda da mortalidade infantil nos últimos decênios no Brasil, com maior aceleração nas regiōes mais desenvolvidas e na área urbana, quando comparada com a rural.

Mediante essa controvérsia, consideramos que um outro dado apresentado pelo IBGE deva ser analisado nessa situação: “... é fundamental a existência de sistemas de informação mais adequados sobre morbi-mortalidade, que funcionem como apoio logístico, tanto na orientação de intervençōes junto aos segmentos populacionais, como na avaliação do alcance das políticas e programas adotados".

Apesar da controvérsia em relação a elevação ou redução dos coeficientes de morbi-mortalidade infantil, consideramos que de qualquer forma, a saúde da criança não tem recebido a atenção e a importância necessárias. Em termos de programação e prática efetiva, de abrangência nacional, o programa de 1984 ainda não foi substituído.

Apesar da $8^{\mathrm{a}}$ Conferência Nacional de Saúde - C.N.S. - ter formulado uma concepção mais ampliada de saúde, “... resultante das condiçōes de alimentação, habitação, educação, renda, meio ambiente, trabalho, transporte, emprego, lazer, liberdade, acesso e posse da terra e acesso a serviços de saúde", COSTA et al (1989), colocam que não se conseguiu avançar na prática profissional. Para a efetivação dessa prática, seria segundo os autores, indispensável entender as limitaçōes de várias 
ordens que se apresentam, quer ao nivel dos serviços de saúde, quer no plano dos direitos de cidadania.

Entendemos, pelo que foi colocado, que a luta por uma melhor assistência à saúde da criança deve estar aliada à luta por melhores condiçōes de vida. Fatores como renda familiar, habitação, saneamento básico, educação básica, interferem diretamente na qualidade de vida e, conseqüentemente, na qualidade de saúde da população.

Notamos que há falta de uma política de saúde inserida em uma política social mais abrangente que tenha como meta a promoção da saúde e não só sua recuperação e reabilitação.

O relatório final da $8^{\mathrm{a}}$ C.N.S. (1986), coloca claramente que não devemos confundir direito à saúde com o direito aos serviços de saúde ou mesmo com o direito à assistência médica, pois a saúde, independentemente de qualquer definição que lhe possa ser atribuída, é produto das condiçōes objetivas da existência. Dentro desse entendimento, "direito à saúde" não corresponderia a uma noção básica exclusiva do processo setorial de formulação de políticas de saúde, mas a um elo integrador que teria que permear todas as políticas sociais do Estado e balizar a elaboração e a implantação das políticas econômicas.

O direito à saúde, assegurado na Constituição, no Estatuto da Criança e do Adolescente, em reuniōes internacionais de altas cúpulas, promulgada e defendida por vários órgãos e governos, ainda encontra-se à nível de discurso. Acreditamos que enquanto a criança não for compreendida e respeitada em sua cidadania, nenhuma programação, restrita em suas definiçōes, alcançará seus objetivos.

DUPAS, G. Considerations on child's state of health. Rev. Esc. Enf. USP., v. 27, n. 3, p. 362-71, dec. 1993.

Health indicators like child morbility and death rates should be analysed in order to set up priorities for the assistence to the child's health. Specialized publications point out that the death rate of children under 5 (TMM 5) has been rising up lately due mainly to food shortage (the lack of nutrients) and infections diseases, in spite of the establishment of programs and goals constitutes with the objective of decreasing this incidence.

UNITERMS: Child's health. Child. Morbi-death rates.

\section{REFERÊNCIAS BIBLIOGRÁFICAS}

ALVARENGA, A.T. Reflexōes acerca da abordagem sociológica do crescimento e do desenvolvimento da criança no campo da saúde pública: aspectos teórico metodológico. Rev.Bras.Cresc.Des.Hum., v.1, n.2, p. 51-64, 1991. 
ASPECTOS generales de la mortalidad en las Américas. Bol. Epidemiol., v.8, n.5/6, p. $1-7,1987$.

BENÍCIO, M.H.D.A. et al. Perfil de morbidade e padrão de utilização de serviços de saúde das crianças brasileiras menores de cinco anos-1989. In: PERFIL estatístico de crianças e màes no Brasil. Rio de Janeiro, UNICEF/INAM, 1992.

BRASIL. Ministério da Saúde. Assistência integral à saúde da criança: açōes básicas. Brasília, Centro de Documentação do Ministério da Saúde, 1984 (Textos Básicos de Saúde, 7).

CAMPESTRINI, S. Alojamento conjunto e incentivo à amamentaçào. Curitiba, Universidade Católica do Paraná, 1983.

CONFERENCIA NACIONAL DE SAÚDE, 8, Brasília, 1986. Anais. Brasília, Centro de Documentação do Ministério da Saúde, 1987.

COSTA, N.R. et al. Demandas populares, politicas públicas e saúde. Petrópolis, Vozes/ABRASCO, 1989.

FERREIRA, J.V. et al. Alguns aspectos do ensino de pediatria no Brasil. J.Pediat., v.56, n.5, p. 332.4, 1984 .

FUNDAÇĀO INSTITUTO BRASILEIRO DE GEOGRAFIA E ESTATÍSTICA - IBGE. Crianças \& Adolescentes: indicadores sociais. Rio de Janeiro, 1992, v.4.

FUNDO DAS NAÇÕES UNIDAS PARA A INFÂNCIA - UNICEF. Situação mundial da infância 1989. Brasilia, 1989.

FUNDO DAS NAÇŌES UNIDAS PARA A INFÂNCIA - UNICEF. Situaçāo mundial da infância 1990. Brasília, 1990.

FUNDO DAS NAÇŐES UNIDAS PARA A INFÂNCLA - UNICEF. Situaçāo mundial da infância 1991. Brasilia, 1991.

GODOY, I.L. O que o enfermeiro faz pela saúde da criança no Brasil. Rev.Paul.Enf., v.4, n.2, p.82-4, 1984 .

MONTEIRO, C.A. et al. Estudo das condiçōes de saúde das crianças do município de São Paulo, SP (Brasil), 1984-1985. Rev.Saúde Públ., v.20, n.6, p.446-53, 1986.

Saúde e nutrição das crianças de Sảo Paulo: diagnóstico, contrastes sociais e tendências. S.Paulo, HUCITEC/EDUSP, 1988.

MORA, G.E. et al. Mortalidade infantil. J.Bras.Med., v.50, n.3, p. 67-82, 1986.

NAKAMAE, D.D. Novos caminhos da enfermagem: por mudanças no ensino e na prática da profissão. S.Paulo, Cortez, 1987.

ORGANIZAÇĀO PANAMERICANA DE SAÚDE. Cuarenta anos de progresso en la salud. Bol.Ofic.Sanit.Panam., v.104, n.4, p. 399-401, 1988.

PAIM, S. et al. Mortalidade infantil proporcional, Salvador, Bahia, Brasil. Bol.Ofic.Sanit.Panam., v.103, n.2, p.113-22, 1987. 
ROCHA, S.M.M. Puericultura e enfermagem. São Paulo, Cortez, 1987.

ROCHA, S.M.M. et al. Assistència de enfermagem à criança no Brasil: estudo histórico e reflexōes sobre as tendências atuais. Ribeirão Preto, s.d./mimeografado/

SAAD, P.M. Mortalidade infantil por causas no Estado de São Paulo (BR) em 1983. Rev.Saúde Públ., v.20, n.6, p.481-8, 1986.

WILLIANSON, R.R. La salud y la nutricion infantil en los paises en desarrollo. Bol.Ofic.Sanit.Panam., v.107, n.4, p. 357-62, 1989. 